\title{
Investigation of the neutron-gamma ray discrimination performance at low neutron energy of a solution-grown stilbene scintillator
}

\author{
Augusto Di Chicco ${ }^{1,3,{ }^{*}}$, Michael Petit ${ }^{2}$, Robert Jacqmin ${ }^{1}$, Vincent Gressier ${ }^{2}$, Brian Stout ${ }^{3}$ \\ ${ }^{I}$ CEA Cadarache, DEN/DER, 13108 Saint-Paul-lez-Durance, France. \\ ${ }^{2} I R S N$ Cadarache, SDOS/LMDN, 13108 Saint-Paul-lez-Durance, France. \\ ${ }^{3}$ Aix-Marseille Université, Institut Fresnel - UMR 7249, 13397 Marseille, France.
}

\begin{abstract}
The results of experiments performed with a $\emptyset 25$ x $25 \mathrm{~mm}$ solution-grown stilbene crystal in mono-energetic neutron fields in the 80 -to- $230 \mathrm{keV}$ energy range are presented. The goal of the measurements, performed at the AMANDE facility, was to explore the capabilities of this organic scintillator to measure neutrons at the lowest possible energy with good pulse shape discrimination (PSD). The time of flight (TOF) technique was used in order to help with the neutron-gamma discrimination. The data are collected via a programmable digital acquisition (DAQ) system CAEN DT7530 using the software CoMPASS with the charge comparison method (CCM). The data are analysed using post-processing codes developed in the ROOT environment. The results show that the stilbene detector has discrimination capabilities for energies as low as $80 \mathrm{keV}$.
\end{abstract}

Keywords - PSD, Organic scintillators, Solution-grown stilbene, Mono-energetic neutrons, Time of flight, digital acquisition system, AMANDE facility.

\section{INTRODUCTION}

For several nuclear applications such as neutron spectrum measurements in zero-power experimental nuclear reactors, nuclear material detection, fusion diagnostics etc., there is a need for fast neutron detectors that can cover a wide energy range, especially from a few $10 \mathrm{keV}$ 's to $10 \mathrm{MeV}$, and that can operate in mixed neutron-gamma radiation fields. These neutron spectrometers must have a good energy resolution $(<10 \%)$, high detection efficiency, be compact and easy to handle, while not being too sensitive to thermal neutrons. Organic scintillators have many of these desirable characteristics, their performance being dependent on the type of scintillating material used: liquid, plastic or crystal. However, organic scintillators are sensitive to gamma rays. Consequently, pulse shape discrimination (PSD) techniques have to be applied to the output signals given by the photomultiplier (PMT) to enable neutron-gamma discrimination. For energies below $1 \mathrm{MeV}$, the discrimination becomes increasingly difficult due to the weakness of the signals given by the light produced by the scintillators. The organic scintillators commonly used as references for neutron spectroscopy in mixed fields are liquid scintillators such as the BC501A and NE213 [1], but these devices have limitations in terms of energy performance (poor discrimination below $1 \mathrm{MeV}$ ) and operational security (fire hazard). In recent years, organic scintillator technology has been significantly improved and digital acquisition systems are now able to replace the traditional analogic systems. The main advantages are the reduction of the electronic acquisition chain and the possibility to store each event and its information like time and pulse shapes. In this way, it is possible to perform offline studies. This is much more difficult with analog systems, therefore the signal generally needs to be processed as it is generated. Recently, a new organic scintillator based on a $\varnothing 25$ x $25 \mathrm{~mm}$ solutiongrown stilbene single crystal was developed [2] and tested at $5 \mathrm{MeV}$ and $565 \mathrm{keV}$. However, the available measurements were insufficient for establishing the low neutron-energy threshold below which neutron-gamma discrimination becomes impossible. Our goal is therefore to investigate this threshold in mono-energetic neutrons fields between $80 \mathrm{keV}$ and $230 \mathrm{keV}$.

\section{EXPERIMENTAL DETAILS}

\section{A. The AMANDE facility}

The AMANDE facility of the Institute for radiological protection and nuclear safety (IRSN) at Cadarache is able to produce continuous or pulsed monoenergetic neutrons from $\sim 2 \mathrm{keV}$ up to $20 \mathrm{MeV}$ [3]. The neutron field for this work was produced using the ${ }^{7} \mathrm{Li}(\mathrm{p}, \mathrm{n})$ reaction with a proton beam of $2067 \mathrm{keV}$ energy and $1 \mu \mathrm{A}$ intensity sent on a LiF target of $107 \mu \mathrm{g} / \mathrm{cm}^{2}$ thickness. The neutron energy at $0^{\circ}$, i.e. in the proton beam direction is $304 \mathrm{keV}$, but more neutron energies are actually accessible by varying the detection angle as shown in Table 1 . The experimental area $\left(20 \times 20 \times 16 \mathrm{~m}^{3}\right)$, is designed so as to limit neutron returns by scattering on the walls. 


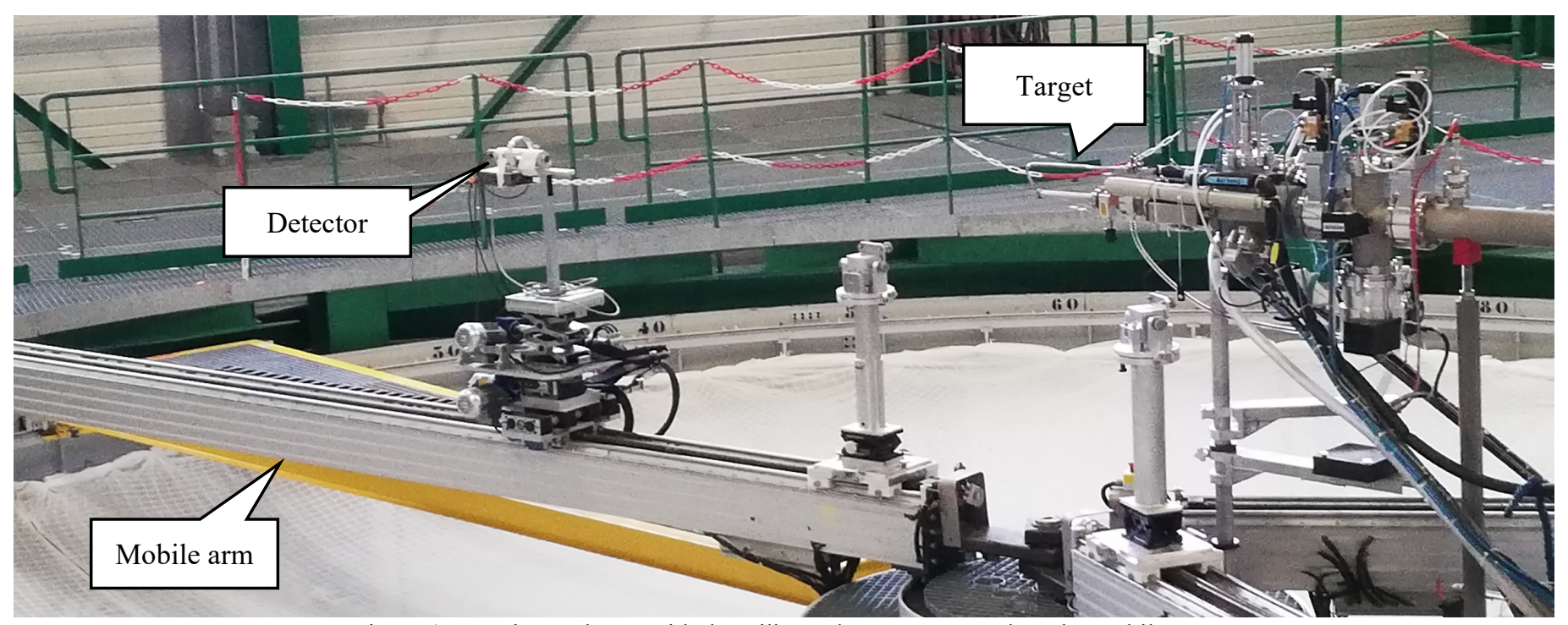

Figure 1 Experimental area with the stilbene detector mounted on the mobile arm

Table 1 Angles relative to proton beam direction and corresponding neutron energies

\begin{tabular}{|c|c|c|}
\hline \hline Angle & Neutron energies & $\begin{array}{c}\text { Monoenergetic } \\
\text { peak FWHM }\end{array}$ \\
\hline $\mathbf{4 5}^{\circ}$ & $223 \mathrm{keV}$ & $7 \mathrm{keV}$ \\
$\mathbf{6 0}^{\circ}$ & $179 \mathrm{keV}$ & $6 \mathrm{keV}$ \\
$\mathbf{8 0}^{\circ}$ & $125 \mathrm{keV}$ & $5 \mathrm{keV}$ \\
$\mathbf{1 0 0}^{\circ}$ & $84 \mathrm{keV}$ & $5 \mathrm{keV}$ \\
\hline
\end{tabular}

Moreover, the presence of a fully-automated transport system can place the detectors at a distance of up to $6 \mathrm{~m}$ from the target, and at any angle between $0^{\circ}$ and $\pm 150^{\circ}$. The time-offlight measurements relies on a pick-up ring placed in the beam at $1 \mathrm{~m}$ in front of the target. The ion beam pulsation is performed using a chopper-buncher system [3b] allowing pulse width around 2 ns.

\section{B. Stilbene Organic Scintillator}

The solution-grown single crystal of stilbene was produced by InradOptics [4], coupled with a photomultiplier ET 9214B of the ET Enterprise [5]. The whole detector was assembled by SCIONIX [6]. The stilbene is encapsulated in an aluminium canister and is optically coupled to the photomultiplier, which is surrounded by a solid mu-metal shield. When an ionizing particle interacts with the scintillator, light is produced. The signals due to incoming gamma rays are different from those produced by neutrons. Gamma-ray interaction leads to electrons and a low-density charge deposit, which excites atoms principally to singlet states. The relaxation times of excited singlets states are very short (less than $10 \mathrm{~ns}$ ) and the related light production is called prompt fluorescence. Neutrons, on the other hand, generate protons via elastic scattering on hydrogen, leading to a high-density charge deposit, which excites atoms to single and triplet states. The lifetime of the excited triplet states is longer (more than $\mu \mathrm{s}$ ) and the corresponding light emission is called delayed [7]. The output signal is taken from the anode of the photomultiplier and the difference in light emission is used to discriminate neutrons (signal with longer decay time due to delayed fluorescence) from photons (prompt fluorescence mainly), as illustrated in Figure 2. However, as the signal amplitude is directly related to the energy of the incident particle (neutron or photon), when neutron energy decreases, the baseline variation (see Section $\mathrm{C}$ below) has a higher impact on the ratio between prompt and delayed fluorescence, and it becomes increasingly difficult to distinguish between the two types of particles.

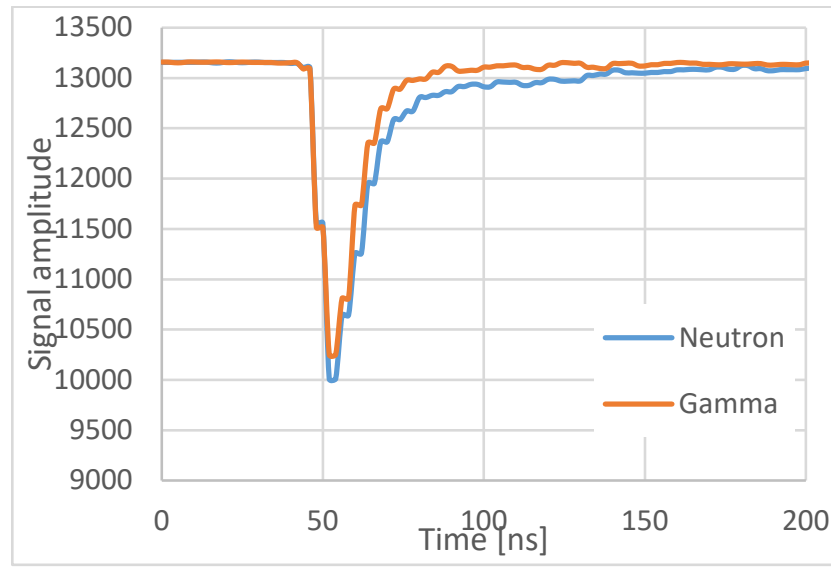

Figure 2 Typical shapes of gamma rays and neutrons

\section{Digital Acquisition System and Charge Comparison Method}

The anode signal from the PMT is directly connected to a DAQ CAEN 5730 [8]. This device has eight channel, 14-bit resolution, $500 \mathrm{MHz}$ sampling rate and a maximum digitizable voltage of $2 \mathrm{~V}$. The online measurements have been performed with the CAEN software CoMPASS [9] using the Digital Pulse Processing - Pulse Shape Discrimination (DPP-PSD) firmware. In order to perform a good online analysis, the DAQ parameters, shown in Figure 3 , have to be optimized according to the type of detector and the experimental situation. 


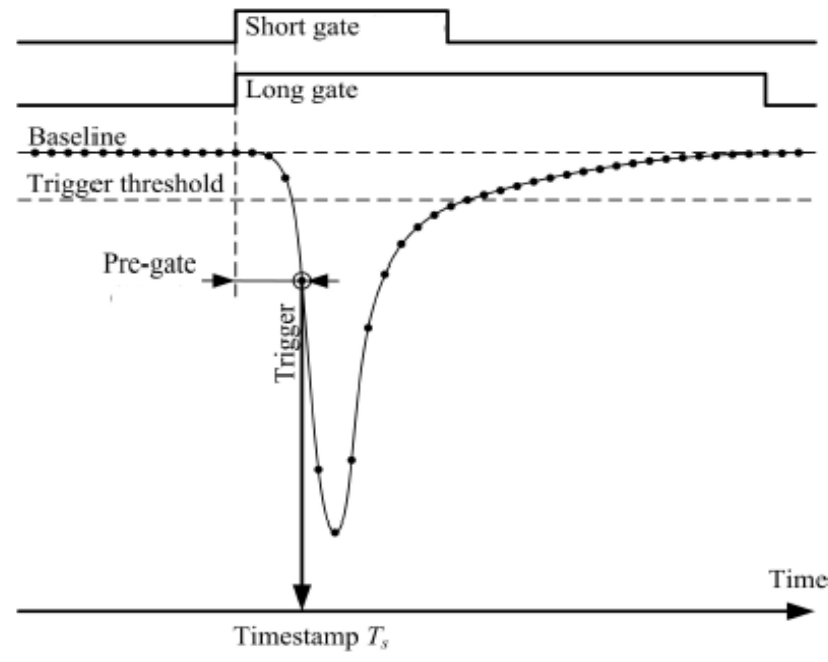

Figure 3 Most relevant parameters for the DPP firmware

Once the recorded signal $i(\mathrm{t})$ exceeds a certain threshold value, the firmware uses the Charge Comparison Method (CCM) to calculate the integrals of the pulses over two different time windows, called "short gate" and "long gate", respectively. These two integral quantities are used in turn to estimate the total light output and the prompt light output. In this way, it is possible to infer a PSD value and discriminate between the detected particles. The equations used to calculate the two integrals are the following:

$$
\begin{aligned}
& Q_{S}=\int_{t_{0}}^{t_{s}} i(t) d t \\
& Q_{L}=\int_{t_{0}}^{t_{L}} i(t) d t
\end{aligned}
$$

where $t_{0}$ is the start time, called pre-gate, for the integrals calculation, while $t_{s}$ and $t_{L}$ correspond respectively to the short and long gates (the optimal values are $34 \mathrm{~ns}$ and $414 \mathrm{~ns}$ for the stilbene). The baseline is determined by averaging over 256 samples, it is subtracted from the signal during the calculation. The PSD is obtained by the following equation:

$$
P S D=\frac{Q_{L}-Q_{S}}{Q_{L}}
$$

The two-dimensional (2D) histogram of the PSD for an AmBe source measured with the stilbene is plotted as a function of $Q_{L}$ (directly related to the energy) in Figure 4 (a). The two areas represent respectively neutrons (high PSD) and gamma rays (low PSD). The PMT was set at $-930 \mathrm{~V}$ in order to avoid saturation and excessive pile-up counts. The data were handled with ROOT [10].

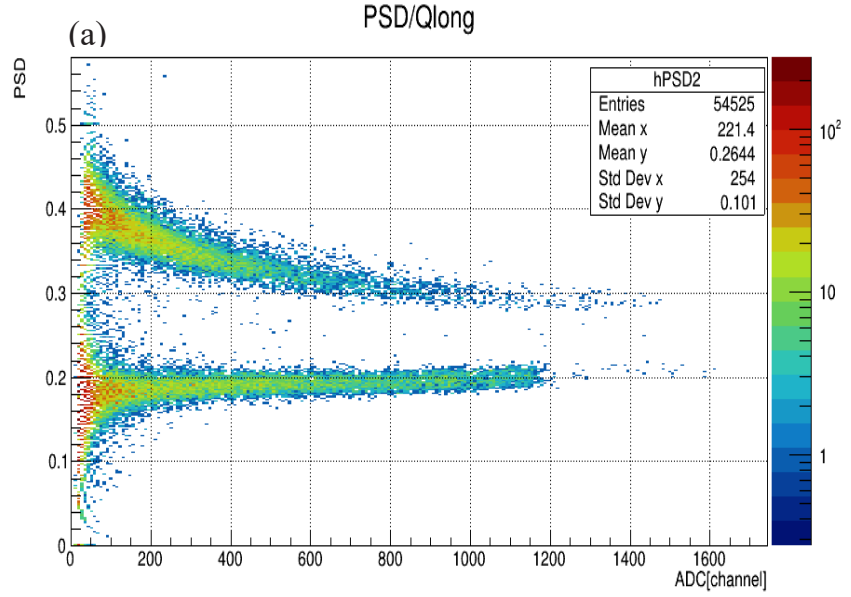

(b) Qlong Total

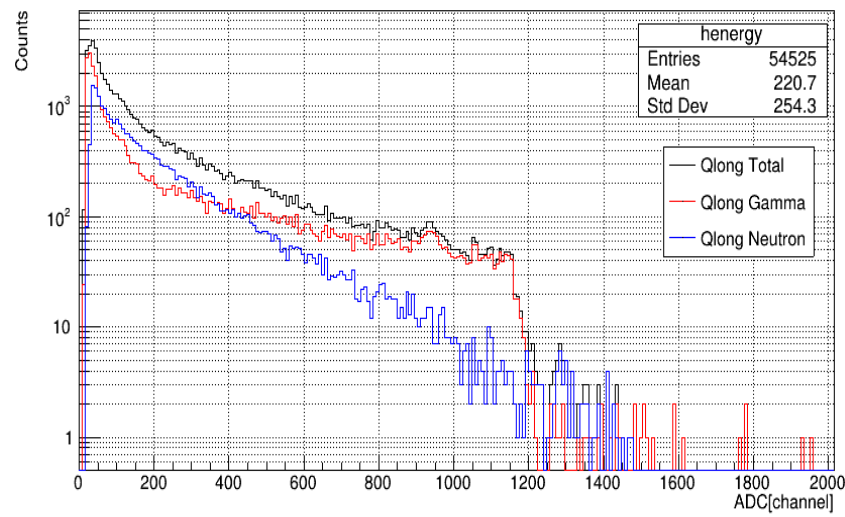

Figure 4 (a) The PSD vs. QL of the AmBe source; (b) Light amplitude for various $Q_{L}$ when applying a cutoff value to the PSD

Figure 4 (b) shows the total light produced (in black) in log scale. The Compton Edge of the $4.438 \mathrm{MeV}$ gamma ray due to the ${ }^{9} \mathrm{Be}(\alpha, n){ }^{12} \mathrm{C}^{*}$ reaction is clearly visible. By considering a cutoff value of 0.26 in the PSD, it is possible to separate the individual contributions of neutrons $(0.26<\mathrm{PSD}<0.6$, in blue) and gamma-rays $(0.06<\operatorname{PSD}<0.26$, in red $)$ to the total signal.

\section{Time of Flight technique}

In the low neutron energy range, the weakness of the signals does not always allow to obtain a clear double-peak PSD profile, and hence setting a cutoff value is difficult. At AMANDE, the time-of-flight technique has been used to help distinguish neutrons from gamma-rays, and to improve the resolution of the PSD. To that aim, the proton beam has been pulsed and the pick-up (PR) ring signal has been connected to the second channel of the DAQ and used as stop signal. The stilbene detector, positioned at a fixed distance $L=1,907$ $\mathrm{m}$ from the target, is connected to the first channel of the digitizer and used as start signal. 


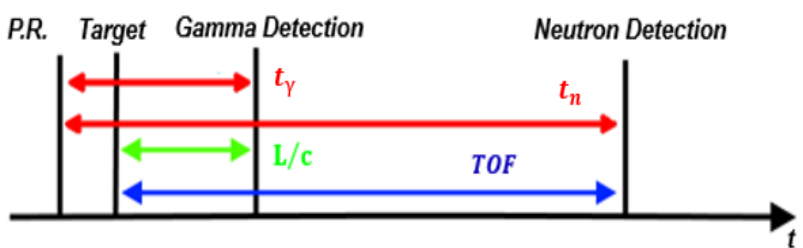

Figure 5 Time of flight scheme for neutron-gamma beams

The DAQ, working in coincidence mode, records the start time of each channel. The difference between these start times is determined with a total resolution of less than $3 \mathrm{~ns}$, for gamma-rays and neutrons simultaneously produced in the target by the pulsed proton beam. The neutron time-of-flight between the target and the detector is simply obtained by writing:

$$
\text { ToF } F_{\text {measured }}=t_{n}-t_{\gamma}+\frac{L}{c}
$$

where $t_{n}$ and $t_{\gamma}$ are the neutron and gamma detection times, respectively, as shown in Figure 5.

\section{EXPERIMENTAL RESULTS}

For the low energy range measurements, the voltage of the PMT was increased up to $-1550 \mathrm{~V}$. However, with this fairlyhigh voltage setting for the PMT, many signals are lost as their amplitude exceed the $2 \mathrm{~V}$ digitizing window, as shown in Figure 6. In addition, there are spurious contributions that give negative values of $\mathrm{Q}_{\mathrm{L}}$ and $\mathrm{Q}_{\mathrm{S}}$, or even some where the total light output value is lower than the prompt one. All these contributions had therefore to be removed from the data analysis.

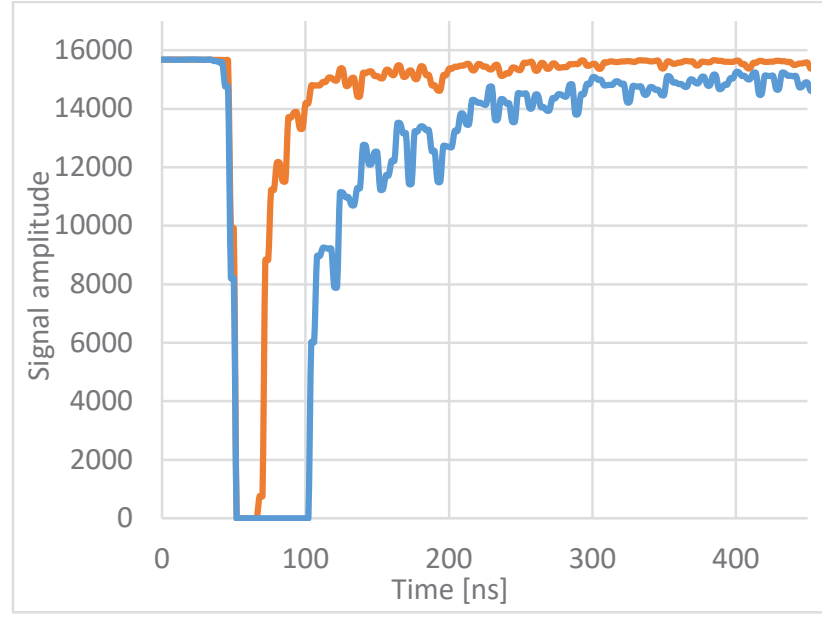

Figure 6 Examples of unusual signals

In Figure 7 (a), the PSD is plotted versus $Q_{L}$ where it is still possible to discriminate the neutrons from the photons. The upper part of the plot (PSD>0.7) corresponds to signals with bad pulse shape that must be eliminated. In Figure 7 (b), the PSD is plotted versus the time difference between the start and stop from the DAQ, in pulse mode. The areas corresponding to the gamma-rays at $100 \mathrm{~ns}$ and to the neutrons at $404 \pm 10 \mathrm{~ns}$ are clearly visible. Below the neutron structure, it is also possible to see the gamma rays produced by neutron interactions in the stilbene crystal.
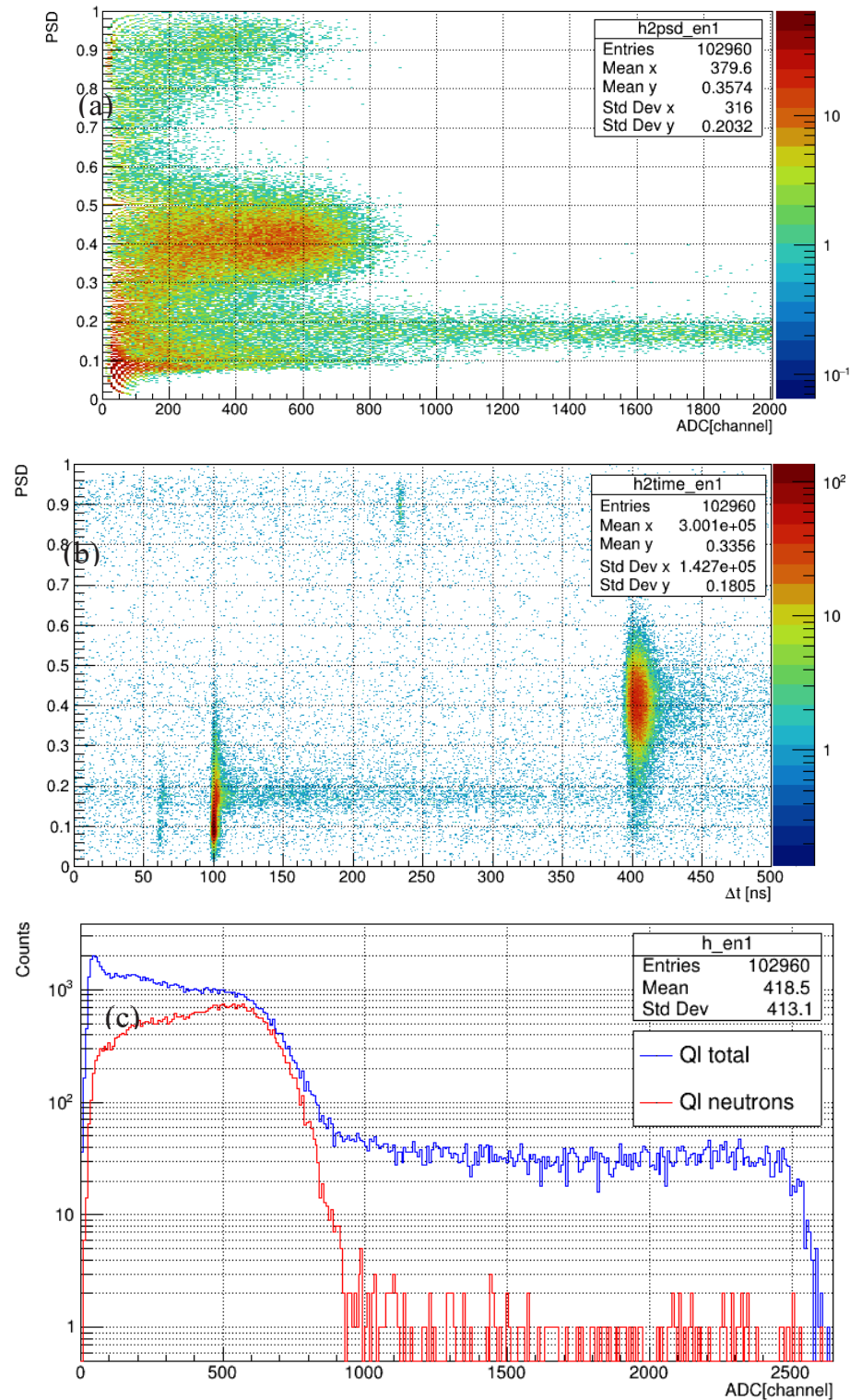

Figure 7 Stilbene data from the DAQ for $230 \mathrm{keV}$ neutrons

In Figure 7 (c), the total pulse height spectra (in blue) is plotted, as well as the derived neutron PHS after selecting the neutron contributions in the time interval $390<\Delta \mathrm{t}<425 \mathrm{~ns}$. Figure 8 shows the time distributions recorded in the four neutron fields from Table 1. The monoenergetic peak is clearly visible even at $84 \mathrm{keV}$, the lowest energy. The corresponding recoil proton energy distributions were obtained by selecting only the events corresponding to the monoenergetic neutrons in the time interval, as shown in Figure 9. These distributions will be used to validate upcoming calculated responses of the stilbene scintillator. 


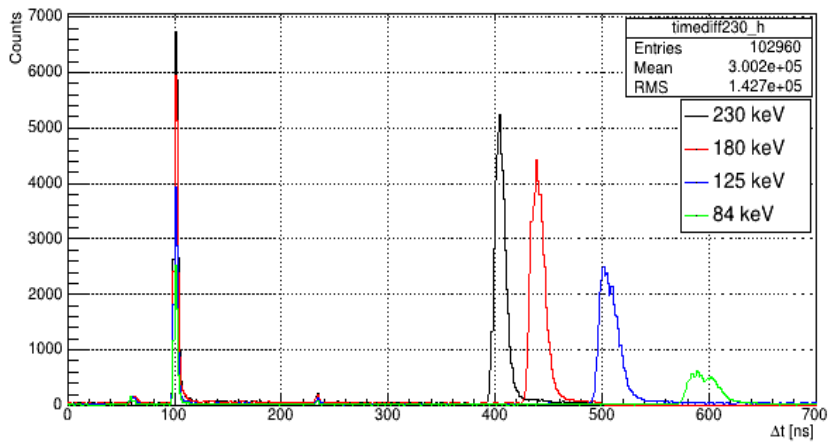

Figure 8 Time of flight distributions for the stilbene

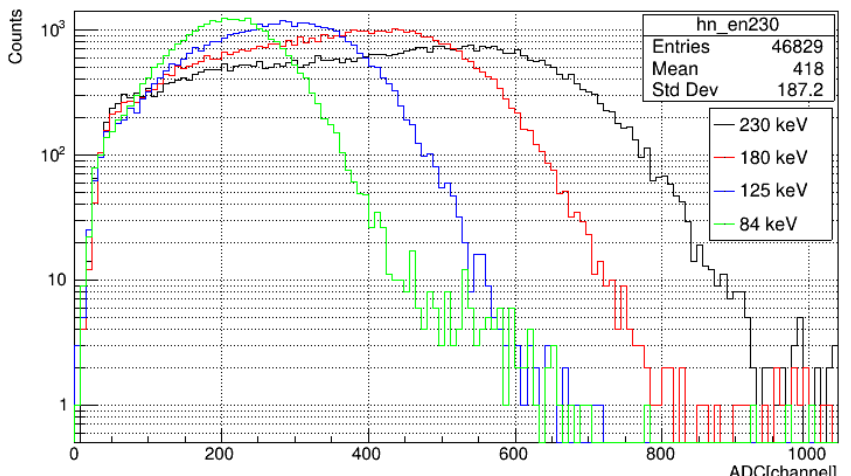

Figure 9 Proton recoil spectra

\section{CONCLUSIONS}

In this article, the capability of an organic scintillator based on a $\varnothing 25 \times 25 \mathrm{~mm}$ solution-grown stilbene single crystal to measure neutrons with energies below $100 \mathrm{keV}$ has been tested at the AMANDE facility, with the help of the time-offlight technique to improve the PSD analyses. The results show that, even at such "low" energies, the stilbene detector still has some neutron-gamma discrimination capabilities, which is remarkable. Upcoming work will focus on the study of individual pulse shapes, so as to have more precise criteria to distinguish neutrons and gamma rays. In addition, the crystal anisotropy will be studied in this energy range as it can significantly influence the detector response. The next step of these studies will be to calculate the stilbene detector response matrix with Monte Carlo codes and to compare it with the experimental data.

\section{ACKNOWLEDGMENT}

The authors thank the entire AMANDE team of IRSN, especially Dr. Richard Babut, for his collaboration and invaluable assistance during the experiments.

\section{REFERENCES}

[1] H. Klein, "Neutron spectrometry in mixed fields: NE213/BC501A liquid scintillation spectrometers," Radiat. Prot. Dosimetry, vol. 107, no. 1-3, pp. 95-109, Nov. 2003.

[2] L. Dioni, V. Gressier, G. Nardin, R. Jacqmin, B. Stout, and M. Sumini, "Tests of a solution-grown stilbene scintillator in mono-energetic neutron beams of 565 $\mathrm{keV}$ and $5 \mathrm{MeV}$," Nucl. Instrum. Methods Phys. Res.
Sect. Accel. Spectrometers Detect. Assoc. Equip., vol. 880, pp. 210-215, Feb. 2018.

[3] V. Gressier et al., "AMANDE: a new facility for monoenergetic neutron fields production between 2 keV and $20 \mathrm{MeV}$," Radiat. Prot. Dosimetry, vol. 110, no. 1-4, pp. 49-52, Aug. 2004.

[4] Inforad, "Production of Stilbene for Fast Neutron Detection."

[5] “9214B Series - ET-Enterprises Ltd.” [Online]. Available: http://etenterprises.com/products/photomultipliers/product/p92 14b-series. [Accessed: 05-May-2019].

[6] "Homepage Scionix.nl * Scionix," Scionix. [Online]. Available: https://scionix.nl/. [Accessed: 05-May2019].

[7] The Theory and Practice of Scintillation Counting. Elsevier, 1964.

[8] “DT5730," CAEN - Tools for Discovery. [Online]. Available: https://www.caen.it/products/dt5730/. [Accessed: 05-May-2019].

[9] "CoMPASS," CAEN - Tools for Discovery. [Online]. Available: https://www.caen.it/products/compass/. [Accessed: 05-May-2019].

[10] "ROOT a Data analysis Framework | ROOT a Data analysis Framework." [Online]. Available: https://root.cern.ch/. [Accessed: 06-May-2019]. 